\title{
Crecimiento de niños "normales" de la ciudad de Córdoba (Argentina), en el primer año de vida, 1993-1994. Estudio CLACYD
}

\author{
Femando Agrelo M.'; Jacobo Sabulsky L. ${ }^{2}$; Beatriz Lobo M. ${ }^{3}$; Lucía Batrouni K. +; \\ Hebe Goldenhersch S. de Roitter'; Liliana Villafañe R. '; Carmen Mongiano N.'; \\ Silvina Berra R. ${ }^{4}$ : Móniça Chesta B. ${ }^{6}$
}

\begin{abstract}
Resumen
Este rabajo describe algunos parómetros del crecimiento de nños "noi trales" de la ciudad de Córdaba, Argentina, en el primer año de vido inacimiento, o y 12 meses.'. Se estudió uno cohorle representalivo, ce distintos estrelos sociales y se determinion medias, desvics estándares y perceniles de peso, longilud y perímeto cefálico. Los valores locales se compararon con un estudio de la cudad de la Plora (Ágentina) y NCHS. y con la meta Propuesla por OMS caro el añi 2000 . Los resulicidos demuestran que los niños corócbeses al nacer pescn, miden y tienen perimeto cetálico mayor que las niñas $1 p<0,003, p<0,001$ y ns respeclivomentel y que a los o y 12 meses esto se mantrene $[0<0,001$ en todos $\mid$. Al comparar los valores por estrako sacral, el peso sólo lue significolivomente mayor en niñas de estratc social aliomedio al nacer ip < 0,02], y a longitud del estrato alto-medic en ambos sexos fue mayor al nacer y al año inirios: $p<0,02$ y $p<0,03$; varones $p<0,05$ y $p<0,03$, respectivamenilal. Al comparar con lablas de la tato y NCHs encontramos que a nacimiento las tres pobaciones sen similares, sin embargo a los o meses los niños y niñas de Córdobo tienen mayor peso y longitud, y a los 12 meses menor peso y longitud. mostando un perfil ce crecimiento distinto. Las meas propuestas por OMS para el año 2000 son alcanzadas en peso y eri longilud para ambos sexas y en tocos tos tiempos esiudiados, excepto la longitud en varones o los 12 meses. Las tablas que se presentar pueden ser utilizadas con lines de inveshgacion, para velorar su adecuación a las caraclerishacas del cecimienlo de los niños cordobeses, de arras regiones de Argentina u atros países con simi ares onlecedentes genético-ambienteles.
\end{abstract}

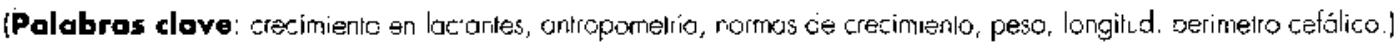

\section{Growth of normal children from Córdoba (Argentina) in their first year of life, 1993-1994. CLACYD Research}

This article destribes some growth parameters of "normol" children Fom the city of Cordoba, Argentuno, in their first year of lie (3i birth, 6 and 12 months). A resresentative cohor from different social levels was studieo. Anthropometric examinations were corried out, occording to standardized methods al cges previously noled. Means, standord ceviations and percenliles of weight, length and heco circurrterence are reporleo. Mcles showed nigrer volues tor weight, height and head circumference then femoles cr the newborn period and along their first yeo of life. Girls from

1. División de Crecimiento y Desarollo, Departamento de Malemidad e Infancia, Ministerio de Salud de la Provincia de Córdoba.

2. Director del Estudio CLACYD. Escuela de Nutrición, Facultad de Ciencias Médicas, Universidad Nacional de Córdoba.

3. Licenciada en Servicio Social. División de Crecimiento y Desarrallo. Departamento de Maternidad e Infancia. Ministerio de Salud de la Provincia de Córdoba.

4. Licenciada en Nutrición. Escuela de Nutrición. Facultad de Ciencias Médicas, Ĺniversidad Nacional de Cordoba.

5. Facultad de Ciencias Económicas, Universidad Nacional de Córdoba.

6. Licenciada en Nutrición. Becaria del Consejo de Investigaciones Científicas y Tecnológicas de la Provincia de Córdoba (CONICOR)

Estudio CLACYD: Córdoba, Lactancia, Alimentación, Crecimiento y Desarrollo. Subsidios: OPS, CONICOR y SeCyT. Apoyo financiero: Fundación ARCOR. 
the high-mean social level showed higher values for weight al borr $\{\rho<0.02$, and both boys ond girls from this social level showed a higher height value than the other social evels analyzed. Local volues were compared with one study from the ciry of lo plato and NCHS. In orde' to find out if the studied populorion survey reoches the goals from $\mathrm{WHO}$ tor the vear 2000 , percentile 10 of weight and length from Cordobo was compared with percertile 3 from NCHS. It is imiporanl that stiese robles be used in future resecrch in order o evaluate its acequocy to the growth pattern of children from Córdoba, other regions of Argenlina or cther counitries with similar generic and enviramental background?

(Key words: intort growth, anth opomely. grow'th norms, weight, length, heod circumference.)

Con la participación de distintas instituciones de la ciudad de Cordoba, Argentina (Escuela de Nutrición, Universidad Nacional de Córdoba, Dirección de Atención Médica Periférica, Municipalidad de Córdoba, División Crecimiento y Desarrollo. Ministerio de Salud de la Provincia de Cordoba, y Sociedad Argentina de Pediatría (Filial Córdoba), se constituý en 1992, un equipo interdisciplinario de investigación que inicia el Estudio CLACYD (C6rdoba, Lactancia, Alimentación, Crecimiento y Desarrollo). Su propósito central es analizar las características de la alimentación, el crecimiento y desartollo de una cohorte representativa de niños urbanos durante sus primeros cinco años de vida.

Ya hemos publicado ${ }^{1.3}$ algunos resultados sobre características sociodemograficas de la población, prácticas de lactancia, su relación con el origen social de los niños, etc.

Pretendemos ahora analizar los datos del crecimiento físico. La información que aporta al respecto el estudio CLACYD es amplia y compleja debido a las características de] diseño de investigacion (longitudinal) y a la multiplicidad de variables analizadas.

En este trabajo nos proponemos describir algunos parámetros del crecimiento (peso, longitud y perímetro cefálico al nacer, 6 y 12 meses) de niños "normales" de la ciudad de Córdoba en el primer año de vida; comparar los resultados del estudio de Córdoba con las tablas de la ciudad de La Plata y las del Centro de Estadísticas de Salud de Estados Unidos (NCHS) ${ }^{5}$; y evaluar, mediante indicadores antropométricos, la situación de los niños "normales" de Cordoba, respecto a las metas fijadas por la OMS para el aก๊o $2000^{6}$.

Otros aspectos de la investigación (evaluación nutricional, composición corporal, velocidad de crecimiento, talla materna, desarrollo psicosocial, etc.) serán abordados en futuras publicaciones.
Con este trabajo esperamos contribuir a la reflexión acerca de la importancia de los estudios antropométricos locales, realizados según criterios metodológicos aceptados a nivel internacional. Nuestro propósito es que la investigación desarrollada en Córdoba, como todo estudio de crecimiento y desarrollo de una población determinada, tenga valor no sólo como indicador de salud de los individuos que la componen, sino también como estrategia básica para identificar los factores del medio ambiente que pueden incidir de manera desfavorable en distintos grupos de población con similar material genético?

Esta investigación es la continuación de una línea de trabajo que iniciamos en Córdoba en 1972 con el estudio transversal de niños de 4 a 12 años ${ }^{8-1}$.

La comparación de los resultados que aquí presentamos con estudios realizados 4 y a realizarse en otras zonas del país, permitirá llegar a algunas conclusiones acerca de la existencia de perfiles de crecimiento y desarrollo "locales. o regionales".

\section{Material y método}

\section{Población}

Tomando como universo los nifios nacidos en 1993, en todas las instituciones sanitarias con servicios de obstetricia (54 establecimientos de salud públicos y privados), de la ciudad de Córdoba, se seleccionó la muestra registrando todos los nacimientos habidos en un período de 12 días (entre el 10 y el 22 de mayo).

Se aplicaron los siguientes criterios de exclusión: domicilio fuera del radio urbano, peso al nacer inferior a $2500 \mathrm{~g}$. productos de partos inúltiples, inalformaciones congentias mayores y enfermedades que pueden afectar el crecimiento y desarrollo. De los 879 nacimientos re. gistrados, se excluyeron 158 dados los criterios mencionados $y 12$ porque la madre rehusó participar en la investigación. La muestra inicial quedó conforınada por lo tanto con 709 casos. A los fines del presente trabajo se incorpo- 
raron sólo para el análisis de los datos antropométricos del nacimiento, a todos los riños nacidos a término, aun aquellos que pesaban menos de $2500 \mathrm{~g}$, con lo cual la muestra inicial del estudio antropomśtrico quedo integrada con 731 casos.

Los datos del estudio antropométrico del nacimiento fuecon tomados de los registros de las instituciones donde nacieron los niños y los de los 6 y 12 meses de edad mediante exámenes antropométricos realizados en los domicilios.

El examen antropométrico de los 6 meses de edad se realizó sobre una submuestra de 230 niños seleccionados por muestreo aleatorio simple a partir de la muestra inicial: las mediciones anlropométricas se efectuaron a 202 ninos. es decit al $88.3 \%$ de la submuestra seleccionada. Fueron limitaciones de carácler opcrativo las que determinaron el achicamiento de la muestra en esta toma de datos. En el estudio antropomérrico del año de edad, se incluyś a los 650 casos entrevistados al mes de vida en la primera encuesta domiciliarial-3, se efeciuaron mediciones antropométricas a 571 nifros, es decir al 87,8 del total previsto y a los fines de esta presentación, sólo se analizan los datos antroponétricos obtenidos dentro de los \pm 15 dias de la fecha de] cumpleaños (484 niños).

Como uno de los objetivos de esta publicación es describir algunos parámetros del crecimiento de niños normales de la ciodad de Cordoba, se han excluido aquellus niflos que presentaron valores extremos ( 4 casos) 13. $^{3}$.

Se estudiaron finalmente un total de 731 niños al nacer ( 358 varones), a los 6 meses 202 (10l varones) y a los 12 meses 484 niños (233 varones).

\section{Estrategia metodológica}

El Estudio CLACYD es de tipo prospectivo. Se estudia una cohorte de niños nacidos en la cjudad de Cordoba en mayo de 1993.

La población se distribuyó en seis grupos sociales definidos según la ocupación del principal responsable del sustento familiar ${ }^{\sharp}$. Para simplificar el análisis de los datos, en este trabajo se adoptó el reagrupamiento de la muestra en dos estratos sociales, ubicándose $52 \%$ de niños en estratos alto/medios, que corresponden a los estratos I (17\%), II (16\%) y III (19\%); y $48 \%$ en estratos bajo/muy bajos, que corresponden a los estratos IV (14\%), V (12 \%) y VI (22\%). Durante el primer año de la investigación, el desgranamiento de la población estudiada (producido por cambios de domicilio y en menor proporción por rechazo de los padres) no modificó significativamente la distribución original de la población en estratos sociales.

Las mediciones antropométricas estuvieron a cargo de profesionales previamente entrenados en la División Crecimiento y Desarrollo, según la metodología recomendada por el Centro In- ternacional de la Infancia ${ }^{14}$. Antes de iniciar el trabajo de campo se efectuó la estandarización de las mediciones de acuerdo al procedimiento diseñado por Habitch ${ }^{13}$. Se conformaron cuatro equipos de dos personas cada uno: examinador y asistente. Este último es imprescindible en la medición de la longitud para sostener la cabeza del niño en la posición adecuada, ya que los errores de medición cuando colabora la madre pueden ser mayores a lo aceptable $(0,4 \mathrm{~cm}), y$ por lo tanto los datos no serían adecuados para el cálculo de incrementos 16

Los instrumentos utilizados fueron: balanza de palanca para lactantes (CAM MR) de $16 \mathrm{~kg}$ de capacidad con un límite de lectura de $10 \mathrm{~g}$, antropómetro de madera construido según normas establecidas ${ }^{14}$, cinta metálica flexible y calibre de presion constante (Lange). Se registraron los siguientes parámetros: peso, longitud, longitud vertex-isquión, perímetro cefálico, perímetro torácico, circunferencia máxima de brazo, tejido celular subcutáneo del tríceps, tejido celular subcutáneo subescapular, erupción deṇtada, talla materna. En esta presentación se analizan en forma transversal los datos correspondientes a peso, longitud y perímetro cefálico.

El procesamiento de los dalos se realizó con el paquete SPSS (spss/pc +4.0$)^{13}$. Se analizaron las variables independientes peso, longitud y perímetro cefálico (medias, desvíos estándar y distribución percentilar) y se empleó la prueba $T$ para las diferencias de promedios entre estratos. Consideramos una diferencia como estadísticamente significativa cuando la probabilidad de que la misma se produjera por azar resultó inferior a $0,05(p<0,05)$.

\section{Resultados}

En la tabla l se presentan medias, desvios estándar y percentiles de peso, longitud y perímetro cefálico de mujeres y varones al nacimiento, 6 y 12 meses de vida.

A los fines de la comparación por sexo, tomamos como referencia la media de los tres parámetros analizados. El peso de nacimiento de los varones es $100 \mathrm{~g}$ superior al de las mujeres $(p<0,003$ ), y a los 6 y 12 meses los varones pesan 567 y $528 \mathrm{~g}$ más respectivamente que las mujeres $(\mathrm{p}<0,001)$. La longitud al nacer es significativamente mayor en los varones (p < 


\section{Tabla 1}

Medias, desvíos estándar y percentiles de peso, longitud y perímetro cefálico

\begin{tabular}{|c|c|c|c|c|c|c|c|c|c|}
\hline \multirow{2}{*}{ Parámeiros } & \multirow[b]{2}{*}{$\overline{\mathbf{x}}$} & \multirow[b]{2}{*}{ DE } & \multirow[b]{2}{*}{ p3 } & \multicolumn{2}{|c|}{ Mujeres } & \multirow[b]{2}{*}{ p50 } & \multirow[b]{2}{*}{ p 75} & \multirow[b]{2}{*}{ p90 } & \multirow[b]{2}{*}{ p97 } \\
\hline & & & & plo & $\mathrm{p} 25$ & & & & \\
\hline \multicolumn{10}{|l|}{ Nacimiento: } \\
\hline $\begin{array}{l}\text { Peso }(\mathrm{g}) \\
\text { Longitud }(\mathrm{cm}) \\
\text { Perimetro cefálico }(\mathrm{cm})\end{array}$ & $\begin{array}{r}32.56 \\
49,6 \\
34,2\end{array}$ & $\begin{array}{r}418 \\
2,1 \\
1,3\end{array}$ & $\begin{array}{r}2450 \\
45,3 \\
32,0\end{array}$ & $\begin{array}{r}2750 \\
47,0 \\
3,3,0\end{array}$ & $\begin{array}{r}2950 \\
48,5 \\
33,1\end{array}$ & $\begin{array}{r}3270 \\
50,0 \\
34,0\end{array}$ & $\begin{array}{r}3550 \\
51,0 \\
35,0\end{array}$ & $\begin{array}{r}3771 \\
52,0 \\
36,0\end{array}$ & $\begin{array}{r}4089 \\
53,0 \\
36,7\end{array}$ \\
\hline \multicolumn{10}{|l|}{6 meses: } \\
\hline $\begin{array}{l}\text { Peso (g) } \\
\text { Longitud (cm) } \\
\text { Perimetro cefádico (cni) }\end{array}$ & $\begin{array}{r}7515 \\
66,2 \\
42,7\end{array}$ & $\begin{array}{r}792 \\
2,5 \\
1,2\end{array}$ & $\begin{array}{r}6293 \\
62,3 \\
40,1\end{array}$ & $\begin{array}{r}6550 \\
63.0 \\
41.0\end{array}$ & $\begin{array}{r}7010 \\
64,5 \\
42,0\end{array}$ & $\begin{array}{r}7450 \\
66.1 \\
42,9\end{array}$ & $\begin{array}{r}8000 \\
67.8 \\
43.5\end{array}$ & $\begin{array}{r}8516 \\
69.1 \\
44.1\end{array}$ & $\begin{array}{r}9209 \\
71,3 \\
44,9\end{array}$ \\
\hline \multicolumn{10}{|l|}{2 meses: } \\
\hline $\begin{array}{l}\text { Peso (g) } \\
\text { Longitud (cm) } \\
\text { Perímelro cefálico }(\mathrm{cm})\end{array}$ & $\begin{array}{r}9425 \\
73,4 \\
45,4\end{array}$ & $\begin{array}{l}995 \\
2,6 \\
1,3\end{array}$ & $\begin{array}{r}7583 \\
68.1 \\
42.7\end{array}$ & $\begin{array}{r}8150 \\
70.3 \\
43.8\end{array}$ & $\begin{array}{r}8740 \\
71,6 \\
44,6\end{array}$ & $\begin{array}{r}9430 \\
73,5 \\
45,5\end{array}$ & $\begin{array}{r}10050 \\
75.3 \\
46.2\end{array}$ & $\begin{array}{r}10730 \\
76,8 \\
46,9\end{array}$ & $\begin{array}{r}150 \\
78,3 \\
47,9\end{array}$ \\
\hline \multicolumn{10}{|c|}{ Varones } \\
\hline \multicolumn{10}{|l|}{ Nacimiento: } \\
\hline $\begin{array}{l}\text { Peso (g) } \\
\text { Longitud }(\mathrm{cm}) \\
\text { Perimetro cefálico }(\mathrm{cm})\end{array}$ & $\begin{array}{r}3356 \\
50,2 \\
34,9\end{array}$ & $\begin{array}{r}418 \\
2,1 \\
1,4\end{array}$ & $\begin{array}{r}2500 \\
46.0 \\
32,0\end{array}$ & $\begin{array}{r}2850 \\
48,0 \\
33,0\end{array}$ & $\begin{array}{r}3082 \\
49,0 \\
34,0\end{array}$ & $\begin{array}{r}3300 \\
50,0 \\
35,0\end{array}$ & $\begin{array}{r}3650 \\
51.5 \\
36,0\end{array}$ & $\begin{array}{r}3883 \\
53,0 \\
36,5\end{array}$ & $\begin{array}{r}4106 \\
54,0 \\
38,0\end{array}$ \\
\hline \multicolumn{10}{|l|}{6 meses: } \\
\hline $\begin{array}{l}\text { Peso }(\mathrm{g}) \\
\text { Longitud }(\mathrm{cm}) \\
\text { Perímetro cefálico }(\mathrm{c}(\mathrm{n})\end{array}$ & $\begin{array}{r}8082 \\
67,7 \\
43.7\end{array}$ & $\begin{array}{l}8.36 \\
2.6 \\
1.4\end{array}$ & $\begin{array}{r}6331 \\
62,4 \\
40,5\end{array}$ & $\begin{array}{r}7064 \\
64,5 \\
42,0\end{array}$ & $\begin{array}{r}7455 \\
66,0 \\
42,8\end{array}$ & $\begin{array}{r}8050 \\
68,0 \\
43,8\end{array}$ & $\begin{array}{r}850 \overline{5} \\
69,2 \\
44,7\end{array}$ & $\begin{array}{r}214 \\
70,4 \\
45,4\end{array}$ & $\begin{array}{r}10012 \\
73,1 \\
46,6\end{array}$ \\
\hline \multicolumn{10}{|l|}{12 meses: } \\
\hline $\begin{array}{l}\text { Peso (g) } \\
\text { Longitud (cm) } \\
\text { Perímetro cefálico (cm) }\end{array}$ & $\begin{array}{l}9953 \\
74,5 \\
46,5\end{array}$ & $\begin{array}{r}1047 \\
2,8 \\
1,3\end{array}$ & $\begin{array}{r}8050 \\
68,3 \\
43,9\end{array}$ & $\begin{array}{r}8586 \\
70,9 \\
44,8\end{array}$ & $\begin{array}{r}9335 \\
72,7 \\
45,7\end{array}$ & $\begin{array}{r}900 \\
74,6 \\
46,5\end{array}$ & $\begin{array}{r}10595 \\
76,5 \\
47,5\end{array}$ & $\begin{array}{r}11368 \\
78.0 \\
48,3\end{array}$ & $\begin{array}{r}12219 \\
79,5 \\
49,0\end{array}$ \\
\hline
\end{tabular}

Estudio CLACYD, Córdoba 1993/1994.

$0,001)$ y a los 6 meses aventajan a las mujeres en $1,5 \mathrm{~cm}(p<0,001)$ y a los 12 meses la diferencia se reduce a $1,1 \mathrm{~cm}(p<0,001)$. Los niños tienen al nacer un perímetro cefálico $0,7 \mathrm{~cm}$ mayor que las niñas (ns) y a los 6 y 12 meses, las diferencias $(1,0$ y $1,1 \mathrm{~cm}$ respectivamente a favor de los varones) son significativas ( $p<$ 0,001 ).

Las tablas 2 y 3 permiten comparar los resultados del estudio de Cordoba con las tablas de La Plata 417 y el NCHS, en ellas se indican los percentiles 10,50 y 90 de peso y longitud.
A analizar los valores del percentil 50 de peso, vemos que al nacimiento las medianas de las tres poblaciones son similares, siendo la diferencia mayor $(120 \mathrm{~g})$ en varones, al comparar Córdoba (3 $300 \mathrm{~g}$ ) y La Plata (3 $420 \mathrm{~g}$ ). A los 6 meses los valores de peso en Córdoba, son en general entre 200 y 250 g más altos, observándose una diferencia mayor $(500 \mathrm{~g})$ a favor de las niñas de Córdoba respecto a las de La Plata. A los 12 meses las niñas de Córdoba todavía pesan más que las de La Plata (130 g más), pero menos que las del estudio norteamericano 


\section{Tabla 2}

Comparación de los valores de peso de Córdoba con La Plata y NCHS (percentiles 10,50 y $90(\mathrm{~g})$ )

\begin{tabular}{|c|c|c|c|c|c|c|}
\hline & p10 & $\begin{array}{c}\text { Mujeres } \\
\text { p50 }\end{array}$ & p90 & plt & $\begin{array}{c}\text { Varones } \\
\text { p50 }\end{array}$ & p90 \\
\hline \multicolumn{7}{|c|}{ Nacimiento: } \\
\hline $\begin{array}{l}\text { Cordoba } \\
\text { La Plata } \\
\text { NCHS }\end{array}$ & $\begin{array}{l}2750 \\
2720 \\
2580\end{array}$ & $\begin{array}{l}3270 \\
3340 \\
3230\end{array}$ & $\begin{array}{l}3771 \\
3970 \\
3640\end{array}$ & $\begin{array}{l}2850 \\
2770 \\
2780\end{array}$ & $\begin{array}{l}3300 \\
3420 \\
3270\end{array}$ & $\begin{array}{l}3883 \\
4060 \\
3820\end{array}$ \\
\hline \multicolumn{7}{|l|}{6 meses: } \\
\hline $\begin{array}{l}\text { Córdoba } \\
\text { La Plata } \\
\text { NCHS }\end{array}$ & $\begin{array}{l}6550 \\
5830 \\
6120\end{array}$ & $\begin{array}{l}7450 \\
6950 \\
7210\end{array}$ & $\begin{array}{l}8516 \\
8200 \\
8380\end{array}$ & $\begin{array}{l}7064 \\
6620 \\
6610\end{array}$ & $\begin{array}{l}8050 \\
7800 \\
7850\end{array}$ & $\begin{array}{l}9214 \\
9080 \\
9100\end{array}$ \\
\hline \multicolumn{7}{|l|}{12 neses: } \\
\hline $\begin{array}{l}\text { Cordoba } \\
\text { La Plata } \\
\text { NCHS }\end{array}$ & $\begin{array}{l}8150 \\
8000 \\
8190\end{array}$ & $\begin{array}{l}9430 \\
9300 \\
9530\end{array}$ & $\begin{array}{l}10730 \\
10900 \\
10870\end{array}$ & $\begin{array}{l}8586 \\
8770 \\
8340\end{array}$ & $\begin{array}{r}9900 \\
10220 \\
10150\end{array}$ & $\begin{array}{l}11368 \\
11650 \\
11540\end{array}$ \\
\hline
\end{tabular}

Estudio CLACYD, Córdoba 1993/1994.

\section{Tabla 3}

Comparación de los valores de longitud de Córdoba con La Plata y NCHS

(percentiles 10, 50 y $90(\mathrm{~cm})$ )

\begin{tabular}{|c|c|c|c|c|c|c|}
\hline & p10 & $\begin{array}{c}\text { Mujeres } \\
\text { p50 }\end{array}$ & p90 & p10 & $\begin{array}{c}\text { Varones } \\
\text { p50 }\end{array}$ & p90 \\
\hline \multicolumn{7}{|c|}{ Naimiento: } \\
\hline $\begin{array}{l}\text { Córdoba } \\
\text { La Plata } \\
\text { NCHS }\end{array}$ & $\begin{array}{l}47,0 \\
47,7 \\
46,5\end{array}$ & $\begin{array}{l}50,0 \\
50,0 \\
49.9\end{array}$ & $\begin{array}{l}52,0 \\
53,0 \\
52,9\end{array}$ & $\begin{array}{l}48.0 \\
48.3 \\
47,5\end{array}$ & $\begin{array}{l}50,0 \\
50.6 \\
50,5\end{array}$ & $\begin{array}{l}53.0 \\
53.0 \\
53.5\end{array}$ \\
\hline \multicolumn{7}{|l|}{6 meses! } \\
\hline $\begin{array}{l}\text { Cordoba } \\
\text { La Plata } \\
\text { NCHS }\end{array}$ & $\begin{array}{l}63,0 \\
62,5 \\
62,6\end{array}$ & $\begin{array}{l}66,1 \\
65,3 \\
65.9\end{array}$ & $\begin{array}{l}69,2 \\
68,9 \\
70,2\end{array}$ & $\begin{array}{l}64,5 \\
64,2 \\
64,4\end{array}$ & $\begin{array}{l}68,0 \\
67,5 \\
67,8\end{array}$ & $\begin{array}{l}70.4 \\
70.8 \\
71,3\end{array}$ \\
\hline \multicolumn{7}{|c|}{12 meses: } \\
\hline $\begin{array}{l}\text { Córdoba } \\
\text { La Plata } \\
\text { NCHS }\end{array}$ & $\begin{array}{l}70.3 \\
70.7 \\
70,8\end{array}$ & $\begin{array}{l}73,5 \\
74,0 \\
74,3\end{array}$ & $\begin{array}{l}76.9 \\
78,2 \\
79,1\end{array}$ & $\begin{array}{l}70,9 \\
72,1 \\
72,8\end{array}$ & $\begin{array}{l}74,6 \\
76,0 \\
76,1\end{array}$ & $\begin{array}{l}78,0 \\
79,8 \\
79,8\end{array}$ \\
\hline
\end{tabular}

Estudio CLACYD, Córdoba 1993/1994.

(100 g). En los varones, al año de edad, los valores de peso de Córdoba son 320 y $250 \mathrm{~g}$ respectivamente más bajos que los correspondien tes a La Plata y el NCHS.

En longitud las medianas al nacer son muy similares en las tres poblaciones. A los 6 meses los valores de Córdoba son algo mayores, aun- que la diferencia no alcanza a $1 \mathrm{~cm}(0,8 \mathrm{~cm}$ entre niñas de Córdoba y La Plata). A los 12 meses, al igual que en el peso, la tendencia se invierte, es decir los niños de Córdoba presentan valores inferiores. La diferencia es importante en los varones, siendo 1,4 y $1,5 \mathrm{~cm}$ menos que las tablas de La Plata y NCHS, respectiva- 
mente (a nivel del percentil 10 la diferencia es de $1,1 \mathrm{~cm}$ respecto a La Plata y $1,9 \mathrm{~cm}$ respecto al NCHS).

La tabla 4 muestra las medias de peso y lon. gitud por estrato social. Puede observarse que, en general (exceptuando la longitud de varones al nacimiento), las medias de los estratos alto/ medios son mayores que las correspondientes a los estratos bajo/muy bajos. En lo que respecta a mujeres se registran diferencias significativas por estrato social en el peso de nacimiento $(\mathrm{p}=0,016)$, la longitud al nacer $(\mathrm{p}=0,017)$ y la longitud a los 12 meses $(p=0,024)$. En cuanto a los varones, se observan diferencias significativas por estrato social en la longitud al nacer ( $p$ $=0,042$ ) y longitud a los 12 meses $(p=0,022)$.

En la publicación de la OMS titulada "Preparación de indicadores para vigilar los progresos realizados en el logro de la salud para todos en el año $2000^{\prime \prime}$ se fijan metas para los indicadores del estado de salud y entre ellos se da particular importancia al estado nutricional de los niños. Entre estas metas se señala que el $90 \%$ de los niños tenga valores de peso y talla superiores al percentil 3 de la norma de referencia internacional (NCHS). Para conocer si los niños del estudio de Córdoba alcanzan la meta propuesta por OMS, comparamos el percenti! 10 de Córdoba (por encima del cual está el 90\% de la población) con el percentil 3 de la norma de referencia ${ }^{5}$. Al hacer esta comparacion, vemos en la tabla 5 que el percentil 10 (de peso y longitud) de los niños de Córdoba, de los estratos alto/medios y bajo/muy bajos se encuentra por encima del percentil 3 del NCHS con una excepción: la longitud de varones a los $12 \mathrm{me}$ ses de edad, en que los esiratos bajo/muy bajos y la población global no alcanzan la meta propuesta.

\section{Comentario}

En este trabajo se presentan tablas de tres parámetros antropométricos elaboradas en el marco de un estudio longitudinal. Si bien esto es admisible ${ }^{18}$, contradice de alguna manera las recomendaciones de grupos de expertos que señalan, entre otros requisitos, que los estudios que sirven de base para la confección de este tipo de normas deben ser transversales y realizados sobre muestras amplias de población 19,20.

Et estudio CLACYD no fue diseñado en realidad con el objetivo de construir normas de crecimiento físico, sino con el propósito de analizar las relaciones entre distintos factores asociados al crecimiento y desartollo; de allí su estrategia Iongitudinal. $S$ in embargo, creemos que las características de la muestra seleccionada (su grado de representatividad, tamaño, composición social) hacen que la misma se acerque en gran medida a las recomendaciones vigentes para la construcción de normas de crecimiento. Hecha esta aclaración, presentamos las tablas

Tabla 4

Medias de peso y longitud según estratos sociales

\begin{tabular}{|c|c|c|c|c|c|c|}
\hline $\begin{array}{l}\text { Estratos } \\
\text { sociales }\end{array}$ & $\begin{array}{l}\text { Peso (g) } \\
\text { Al nacer }\end{array}$ & $\begin{array}{c}\text { Mujeres } \\
6 \text { meses }\end{array}$ & 12 meses & Al racer & $\begin{array}{l}\text { Varones } \\
6 \text { meses }\end{array}$ & 12 meses \\
\hline $\begin{array}{l}\text { Alto-medios } \\
\text { Bajo-muy bajos }\end{array}$ & $\begin{array}{c}3309 \\
3199 \\
p<0,02\end{array}$ & $\begin{array}{c}7540 \\
7483 \\
\text { ns }\end{array}$ & $\begin{array}{c}9456 \\
9380 \\
n s\end{array}$ & $\begin{array}{c}3349 \\
3365 \\
\text { ns }\end{array}$ & $\begin{array}{c}8161 \\
8016 \\
\text { ns }\end{array}$ & $\begin{array}{c}10089 \\
9836 \\
\text { ns }\end{array}$ \\
\hline $\begin{array}{l}\text { Estratos } \\
\text { sociales }\end{array}$ & $\begin{array}{l}\text { Longitud (cm) } \\
\text { Al nacer }\end{array}$ & $\begin{array}{l}\text { Mujeres } \\
6 \text { meses }\end{array}$ & 12 meses & Al nacer & $\begin{array}{l}\text { Varones } \\
6 \text { meses }\end{array}$ & 12 meses \\
\hline $\begin{array}{l}\text { Alto-medios } \\
\text { Bajo-muy bajos }\end{array}$ & $\begin{array}{c}49,8 \\
49.3 \\
p<0,02\end{array}$ & $\begin{array}{c}66,4 \\
66,0 \\
\text { DS }\end{array}$ & $\begin{array}{c}73,7 \\
73,0 \\
p<0,03\end{array}$ & $\begin{array}{c}49,9 \\
50,4 \\
p<0.05\end{array}$ & $\begin{array}{c}68,0 \\
67,4 \\
\text { ns }\end{array}$ & $\begin{array}{c}74,9 \\
74,0 \\
p<0,03\end{array}$ \\
\hline
\end{tabular}

Eș1udio CLACYD, Córdoba 1993/1994. 


\section{Tabla 5}

Situación de los niños de Córdoba respecto a las metas fijadas por OMS para el año 2000 (población total y por estratos sociales). Comparación p 10 de Cótdoba con p3 de NCHS

\begin{tabular}{|c|c|c|c|c|c|c|}
\hline $\begin{array}{l}\text { Estratos } \\
\text { sociales }\end{array}$ & $\begin{array}{l}\text { Peso (g) } \\
\text { Al nacer }\end{array}$ & $\begin{array}{l}\text { Mujeres } \\
6 \text { meses }\end{array}$ & 12 meses & Al nacer & $\begin{array}{l}\text { Varones } \\
6 \text { meses }\end{array}$ & 12 meses \\
\hline $\begin{array}{l}\text { Alto-medios } \\
\text { Bajo-muy hajos } \\
\text { Poblac. total } \\
\text { Mela p3 NCHS }\end{array}$ & $\begin{array}{l}2774 \\
2556 \\
2750 \\
2300\end{array}$ & $\begin{array}{l}6.544 \\
65.74 \\
6550 \\
5600\end{array}$ & $\begin{array}{l}8250 \\
8052 \\
8150 \\
7600\end{array}$ & $\begin{array}{l}2874 \\
2830 \\
2850 \\
2500\end{array}$ & $\begin{array}{l}7115 \\
7014 \\
7064 \\
6000\end{array}$ & $\begin{array}{l}8830 \\
8426 \\
8586 \\
8200\end{array}$ \\
\hline $\begin{array}{l}\text { Estratos } \\
\text { sociales }\end{array}$ & $\begin{array}{l}\text { Longitud (cm) } \\
\text { A] nacer }\end{array}$ & $\begin{array}{l}\text { Mujeres } \\
6 \text { meses }\end{array}$ & 12 meses & Al nacer & $\begin{array}{l}\text { Varones } \\
6 \text { meses }\end{array}$ & 12 meses \\
\hline $\begin{array}{l}\text { Alto-medios } \\
\text { Bajo-muy bajos } \\
\text { Poblac. total } \\
\text { Meta f3 NCHS }\end{array}$ & $\begin{array}{l}47,3 \\
46.5 \\
47,0 \\
45,8\end{array}$ & $\begin{array}{l}62,9 \\
63,2 \\
63,0 \\
61,0\end{array}$ & $\begin{array}{l}71,0 \\
70.0 \\
70,3 \\
69.0\end{array}$ & $\begin{array}{l}47,3 \\
48.0 \\
48,0 \\
46,2\end{array}$ & $\begin{array}{l}64,8 \\
63,7 \\
64,5 \\
62,8\end{array}$ & $\begin{array}{l}71,9 \\
70.8 \\
70.9 \\
71,0\end{array}$ \\
\hline
\end{tabular}

Estudio CLACYD, Córdoba 1993/1994.

antropométricas elaboradas en el marco del Estudio CLACYD como expresión de las condiciones genético-ambientales de los niños "normales", menores de un año, de la ciudad de Córdoba. Las mismas podrán ser empleadas con fines de investigación en distintos trabajos para valorar su adecuación a las características del crecimiento físico de los niños cordobeses y de otras poblaciones con similares antecedentes genético-ambientales. Entendemos que la conjunción de estudios locales y regionales contribuirá a la revisión de las normas vigentes y a la elaboración en un futuro próximo de estándares nacionales de crecimiento, representativos de las múltiples realidades de nuestro país ${ }^{2 !}$. Creemos además que la reflexión sobre el tema debería extenderse a aquellos países que no cuentan con estándares nacionales representativos de sus diferentes regiones o unidades sociopolíticas.

Las diferencias observadas por sexo (valores más altos en los varones) en los tres parámetros (peso, longitud, perímetro cefálico) coinciden con los resultados de otros estudios efectuados en el mismo grupo etario.

Respecto a la comparación con las tablas de La Plata y NCHS, los datos muestran a los 6 y 12 meses un perfil distinto de crecimiento en los niños de Córdoba: un mayor crecimiento (tanto en peso como en longitud) en el primer semes- tre de vida, seguido de una desaceleración en el segundo semestre. Este perfil de crecimiento será analizado en una próxima publicación sobre incrementos de peso y longitud.

En algunos casos (peso de mujeres a $\operatorname{los} 6$ meses, Iongitud de varones a los 12 meses), las diferencias con La Plata y NCHS son importantes. Así por ejemplo, el percentil 50 de longitud de varones a los 12 meses se sitúa 1.4 y $1,5 \mathrm{~cm}$ por debajo del de La Plata y NCHS respectivamente. Es interesante destacar que los valores medios de longitud de Córdoba al año de edad son muy similares a los registrados por Habicht $^{22}$ en un análisis de muestras de varias partes del mundo con poblaciones supuestamente bien nutridas de parses desartollados (mujeres: Cordoba $73,4 \mathrm{~cm}$. Habicht $73,3 \mathrm{~cm}$; varones: Cordoba $74,5 \mathrm{~cm}$, Habicht $74,8 \mathrm{~cm}$ ).

Un estudio del CESNI ${ }^{23}$ en niños de 9 a 24 meses, comprobó diferencias en el diagnóstico de malnutrición (según longitud/edad) en los varones, adjudicables al uso de distintas tablas: La Plata $4, \mathrm{SAP}^{17}, \mathrm{NCHS}^{5}$.

En cuanto al perímetro cefálico, si bien no hemos incluido tablas comparativas por razones de espacio, pudo constatarse que los valores de Córdoba (correspondientes a la mediana y -2 $\mathrm{DE} /+2 \mathrm{DE}$ de la mediana), son algo inferiores a los valores de Nelhaus ${ }^{24}$. Al respecto Jordán ${ }^{25}$ 
destaca que "la circunferencia cefálica varía dentro de ciertos límites en los niños de diferentes grupos étnicos, desarrollo socioeconómico y localidad urbana o rural" y recuerda que Meredith ${ }^{26}$ argumentó que los patrones de Nelhaus no eran aplicables a la generalidad de la especie humana.

Los resultados de la tabla 4 son coincidentes con múltiples trabajos que, en el campo de la antropometría nutricional, relacionan la longitud más que el peso, con las condiciones sociales globales de la población ${ }^{27}$. 28. La falta de asociación con el estrato social a los seis meses de vida puede deberse al insuficiente tamano muestral que, a esa edad, es de aproximadamente 50 niños por sexo en cada estrato social.

La tabla 5 muestra que la meta propuesta por OMS para el año 2000 es alcanzada en peso y longitud en todos los estratos y tiempos medidos, excepto por los varones al año de edad en lo que respecta a longitud, edad en la que sólo los estratos sociales alto-medios logran superarla. En la tabla sólo se registran, por razones de espacio, los datos correspondientes a los estratos sociales agrupados. Al analizar los resultados según los seís estratos definidos originalmente en el Estudio CLACYD, se destaca el hecho de que el percentil 10 de longitud (en varones de 12 meses) correspondiente al estrato 6 (trabajadores temporarios), alcanza el valor de $70 \mathrm{~cm}$, es decir se encuentra $1 \mathrm{~cm}$ por debajo de la meta de OMS $(71 \mathrm{~cm})$ y prácticamente $2 \mathrm{~cm}$ por debajo del percentil 10 de los estratos altomedios $(71,9 \mathrm{~cm})$. En síntesis, las diferencias sociales no sólo se expresan en los valores promedio, sino también en el logro de metas mínimas de crecimiento.

Queda finalmente un interrogante: ¿Por qué los valores de Córdoba (en lo que respecta a la longitud de varones al año de edad) son tan distantes de las normas del NCHS y tan cercanos a los publicados por Habitch ${ }^{22}$ en un estudio sobre poblaciones supuestamente bien nutridas de países desarrollados? Sabemos que las tablas del NCHS son propuestas como normas de referencia a nivel internacional para comparar la situación de distintas poblaciones y no como estándares para uso clínico en el niño individual. Ahora bien, iconstituyen un adecuado patrón de referencia para valorar la brecha que separa a poblaciones más desfavorecidas de otras con altos niveles de salud y nutrición?
Una publicación del grupo de trabajo de OMS en Crecimiento Infantil ${ }^{29}$ concluye que las tablas NCHS-OMS son inadecuadas para la evaIuación de los niños amamantados. ¿Ha llegado el momento de confeccionar una nueva norma de referencia basada en una muestra de población más diversificada racial y étnicamente, que incluya además a niños alimentados según las recomendaciones de OMS? Esta parece ser la orientación actual del grupo de trabajo sobre crecimiento infantil de OMS $^{29,} 30$.

\section{Agradecimientos}

Los autores desean expresar su agradecimiento a todos los profesionales que participaron en la recolección y tratamiento de datos: inédicas Abalos $M$. de los A. Frías C. Gea G.. Martínez Catalán A., y licenciados Cossani E., Monterovi C., Eppens ME., Peano ME., Martina D., Salcedo M., Zamora \$. y Toledo S. Además agradecen a la Licenciada Noemí Chotro F, y al Dr. Cados F. González A.. por In dedicacion puesta en la traducción del resumen a] inglés.

\section{Referencias}

I. Sabulsky $J_{1}$ Batrouni L. Carballo R. et al: Alimentación en el primer mes de vida, por estratos sociales, Córdoba, Argentina. Bol of Sanit Panam 1995; 1 19: 15-27.

2. Quiroga D. Sabutsky J. Batrouni L. ef al: Perfiles de iactancia, alimentacion, crecimiento y desarrollo en los primeros años de vida. Estudio CLACYD. Informe preliminar: Perfiles de lactancia hasta el mes de edad. Factores asociados. Salud Infantil 199\$; 4: 5-10.

3. Sabuisky J, Batrouni L, Quiroga D, et al: Perfiles de lactancia al mes de vida. Estudio CLACYD, 1993 Arch Arg Pediatr 1995; 93: 151-157.

4. Cusminsky $M$. Castro $E$, Lejarraga $H$, et al: Tablas norinales de peso, estatura y perímetro cefálico desde el nacimiento hasta los doce años de edad. Arch Ary Pediatr 1980: 79: 281-295

5. United States Department of Heahh Education and Welfare Public Healh Service. Health Resources Administration. NCHS Growth Chatts Rockville MD 1976: 76: $1120-1125$.

6. Organización Mundial de la Solud. Preparación de indicadores para vigilar los progresos realizados en el logro de la salud para todos en el 2000 . Serie Salud para todos. Ginebra, 198I, N 4 .

7. Jordan JR: Crecimiento del nifio como indicador de salud. En: Salud Materno Infantil y Atención Primaria en las Américas. Hechos y Tendencias. OMS. Publicación Científica $\mathrm{N}^{\circ}$ 461, 1984: 71-82.

8. Funes Lastra P. Agrelo F. Guina S. et al: Estudio del crecimiento y desarrollo de nipos normales a traves de una muestra representativa. Congreso Internacional de Pediatría XIV, Buenos Aires, 1974: 62-69. 
9. Funes Lastra P. Agreto $F$ Guita $S$. et al: Estudio del crecirniento y desarrollo de niños normales de la ciudad de Córdoba a través de una muestra rcpresentativa. Crecimiento y Desarrollo (Tomo 1). Ed. Universidad Nacional de Córdoba. Córdoba, 1975.

10. Agretn $F$, Saforcuda $E$. Funes Lasina $P$ : Patrones del teiido celular subcutáneo de niños normales de 4 a 12 años de la ciudad de Cordoha. Arch Arg Pediatr 1977; 75: 69.76 .

11. Agrelo F. Funes Lastra P. Cosiamagna N, el al: Circunferencia máxima del brazo. Su utilidad como indicador del estado de nutrición en estudios epidemiológicos. Arch Arg Pediatr 1982: 80; 170.182.

12. Agrelo $F$, Lobo B. Roister $H$ de. Jazán $G$. Villofañe $L$, Funes Lastra P: Estándares de talla sentada para niños de 4 a 12 años de la cjudad de Córdoba. Arch Arg Pediatr 1995: 93:363-371.

13. Nortsis MJ: Statistical package for the Social Sciences (SPSS/PC+), versión 5.0, Chicago. Illinois $60611,1988$.

14. Faikner F: Croissance el development de l'enfant normal. Une inéthode internationale d'étude. Centre Internationale de L'enfance. Travaux et Documents XIII, París, 1961.

15. Habitch fP: Estandarización de métodos epidemiologicos cuantitativos sobre el terreno. Bol of Sani1 Panam 1974: 76: 375-384.

16. Fonmon SJ: Reference data for assessing growth of infants (Editor's Column). J Pediatr 1991; 119: 415416.

17. Sociedad Argentina de Pediatría. Criterios de diagnóstico y tralamiento: Crecimiento y Desarrollo, 1986

18. Fatkner F: Desartollo humano. Ed. Salvat. Barcelona, 1969: 20.

19. Wateriow JC, Buzina $R$, Keller $W$, et al: The presentation and use of heigth and weigh data for compaling the nutritional staus of groups of children under the age of 10 years. Bull WHO 1977; 55 : 489 498.
20. Organización Panamericana de la Salud. Ficha de crecimiento para uso intemacional en el cuidado de la salud materna e infantil. Guía pam el personal de atención primaria de salud. Publicación Cientifica 409. Washingıon DC, $198 \mathrm{I}$

21. Agrele F: Algunas consideraciones críticas sobre las normas de crecimiento para niños de 0-6 años de la Dirección Nacional de Maternidad e Infancia (Carta al Editor). Arch Arg Pedialr 1987: 85: 342-344.

22. Habicht JP: Height and weight standars for preschool children. How relevant are elhnic difterences in growih potential? Lancet $1974 ; 1: 611-615$.

23. Calvo EB. Carmuega $E$, Gnazo $N$, el al: Evaluación del estado nutricional de la población de nin̄os de 9 a 24 meses de edad residente en los partidos del Gran Buenos Aires. Arch Arg Pediatr 1991; 89: 132-141.

24. Nelhats G: Head circumference from birth to eighteen years. Practical composite internacional and interracial graphs. Pediatrícs 1968:41: 106-114

25. Jordin $J R$ : El lactante de 0 a 2 anos: antropometria y crecimiento. En: Crecimiento y Desarrollo. Hechos y Tendencias. Washington D.C. OMS. Publicación Científica $\mathrm{N}^{\circ}$ 510, 1988: 84-209.

26. Meredith HV: Human head circumference from birth to early manhood: racial, regional and sex comparisons. Growth 1971, 35: 233.

27. Keiler $w$ : Choice of indicators of nutritional slates. En: Evaluation of Nutrition Education in Third World Commnunities. Nestlé Foundation Publication Series, Vol 3, Beat Schürch (Editor) 1982.

28. WHO Working Group: Use and interpretation of anthropometric indicators of motritional status. Bull WHO 1986; 64: 929-941.

29. WHO Workine Group on Infun Growth: The use and interpretation of anthopometry in infants. Bull WHO 1995: 73: $165-174$.

30. WHO Working Group on helum Growth, 1994: An evaluation of infant growth. Nutrition Unit, World Healıh Organization. Géneva, 1994: 41 -43.

\section{AVISO A LOS AUTORES}

Con el objeto de dar prioridad a los trabajos de investigación, en vista de las limitaciones de espacio de la Revista Chilena de Pediatría, el Comité Editorial ha acordado restringir la impresión de casos clínicos a un máximo de dos por cada número. 